\title{
Epitaxial Lead Zirconate Titanate Nanocrystals Obtained by a Self-Patterning Method
}

\author{
I. SZAFRANIAK, ${ }^{*}$ M.-W. CHU, C. HARNAGEA, ${ }^{* *}$ R. SCHOLZ, \\ D. HESSE, and M. ALEXE \\ Max Planck Institute of Microstructure Physics, Weinberg 2, \\ D-06120 Halle, Germany
}

(Received August 8, 2003; in final form January 5, 2004)

Epitaxial lead zirconate titanate nanoislands were obtained by a self-patterning method using the instability of ultrathin films during high-temperature treatments. After high-temperature annealing, the as-deposited film breaks into islands with a narrow size distribution. Nanostructures, as small as $20 \mathrm{~nm}$ lateral size with a height of $9 \mathrm{~nm}$, were epitaxially grown on $\mathrm{Nb}$-doped (001) $\mathrm{SrTiO}_{3}$ substrates. The formation of nanostructures was investigated as a function of the initial film thickness and crystallisation condition. Single-crystal nanoislands were studied by scanning and high-resolution transmission electron microscopy, atomic force microscopy and $\mathrm{X}$-ray diffraction. Ferroelectric switching of several individual islands was investigated by piezoresponse force microscopy.

Keywords: PZT; ferroelectric epitaxial nanosize crystals; self-assembly; piezoresponse-AFM

PACS numbers: 77.84.Dy; 81.16.Dh; 84.07.-b

\section{INTRODUCTION}

Multifunctional ferroelectric materials offer a wide range of useful properties from switchable polarisation that can be applied in memory devices, to piezoelectric and pyroelectric properties used in actuators, transducers and thermal sensors $[1,2]$. However they can not be applied to nanometer scale devices before the influence of the lateral size on physical properties

\footnotetext{
*Present address: Institute of Materials Science and Engineering, Poznan University of Technology, M. Sklodowska Curie 5 Sq., 60-965 Poznan, Poland.

**Present address: INRS-Énergie et Matériaux, 1650 boul. Lionel-Boulet, Varennes QC, J3X 1S2, Canada.
} 
will be clarified. In real system it is difficult to distinguish between the intrinsic and extrinsic size effects caused by crystal imperfections or by surface effects. Therefore, to investigate the true size effect there is a need to have single-crystalline structures with a lateral size that can be tuned in the nanometer range. Recently, there were a few attempts to fabricate ferroelectric nanostructures. However, the lead titanate nanograins obtained by a solution-based dilution technique on platinum-coated silicon had completely random orientation [3-5]. The analysis of the size effect can be rather complicated without any information on the crystal orientation of each grain since ferroelectric and piezoelectric properties are closely connected with crystallographic orientation [6]. A different approach to study nanostructures is based on investigating thin films at their initial growth stage when islands develop [7-9]. A detailed analysis of $\mathrm{PbTiO}_{3}$ and $\mathrm{PZT}$ nanostructures on $\mathrm{Pt}$ coated Si wafers has shown that they do not posses single crystal quality (viz. twin boundaries were observed by TEM) [7, 10], making the investigation of the size effects on those samples even more complicated. Therefore, the best way to study size effects in ferroelectrics is to produce single-crystal, defectfree, mono-domain nanostructures with controlled size and orientation.

In order to fabricate uniform, single-crystal nanosize ferroelectric structures we have applied a self-pattering method based on the instability of ultrathin films during high-temperature treatment. It was previously observed that the preparation of ultrathin epitaxial films by chemical solution deposition (CSD) is hindered by a microstructural instability, i.e. thin films with a thickness below a critical value break up into islands with a narrow size distribution after a high-temperature annealing $[11,12]$. The aim of the present paper is to investigate the influence of the initial film thickness and thermal treatment on island formation. The island morphology and properties were studied by electron microscopy, atomic force microscopy and X-ray diffraction. The ferroelectric behaviour, viz. local switching of polarization by an external field, was verified for individual nanostructures using piezoresponse force microscopy.

\section{EXPERIMENTAL}

$\mathrm{PbZr}_{0.52} \mathrm{Ti}_{0.48} \mathrm{O}_{3}$ (PZT) ultra-thin films of different thickness were obtained by spin-coating a commercial precursor (PZT9906, Chemat Technology, INC.) onto single crystalline niobium doped $\mathrm{SrTiO}_{3}(\mathrm{STO}: \mathrm{Nb})$ (001)-oriented substrates with a Nb concentration of $0.5 \%$ (CrysTec GmbH, Berlin). The initial film thickness was set by dilution of the raw precursor in its solvent (butanol), which ranged from 1:10-1:40, and by adjusting the 
spinning speed from 3000 to $6000 \mathrm{rpm}$. The obtained gel film was dried on a hot plate at $80^{\circ} \mathrm{C}$ for $5 \mathrm{~min}$, pyrolized at $300^{\circ} \mathrm{C}$ for $5 \mathrm{~min}$, and finally crystallised in a lead oxide atmosphere. To study the growth mechanism the crystallisation process was performed by annealing for 5 min$10 \mathrm{~h}$ at different temperatures between $650^{\circ} \mathrm{C}$ and $800^{\circ} \mathrm{C}$. The obtained nanostructures were studied by high-resolution transmission electron microscopy (HRTEM) (JEOL 4010 at $400 \mathrm{kV}$ ), atomic force microscopy (AFM) (Dimension 5000, Digital Instruments), and by X-ray diffraction (XRD) (Philips X'Pert Diffractometer). Piezoresponse force microscopy measurements were performed using an Autoprobe CP Research AFM and a lock-in amplifier (EG\&G Instruments, Model 7260) as described previously $[13,14]$. The ac voltage was applied to the tip mounted on medium-stiff cantilevers of type CSC11b (Micromasch) coated with the hard semiconductor $\mathrm{W}_{2} \mathrm{C}$. The dc voltage for polarisation switching was applied to the bottom electrode of the sample using a computer-controlled Keithley 2400 Source Meter.

\section{RESULTS AND DISCUSSIONS}

The growth of nanostructures was investigated as a function of the initial film thickness. Figure 1(a-c) shows the AFM morphology of the structures obtained after crystallisation at $800^{\circ} \mathrm{C}$ for $1 \mathrm{~h}$ of films with different initial thicknesses. As expected, the microstructural instability generates small single-crystal islands after thermal treatment. If the initial film thickness is just below a critical value, relatively large islands of irregular shape form, as shown in Fig. 1a. Islands have an average height of $15 \mathrm{~nm}$ and a wide distribution in lateral sizes up to a few hundreds nanometers. Thinner layers create more regular islands that are both larger and higher (Fig. 1b). Their height increases up to about $25 \mathrm{~nm}$ due to the contraction in the lateral size, and the conservation of the total volume. The distance between close neighbours increases as well, resulting in a low areal density of the islands. When the initial layers are even thinner the height of islands decreases and their areal density increases (Fig. 1c). The smallest islands obtained from the most diluted precursor have a height of about $9 \mathrm{~nm}$ and lateral dimensions of about $50 \mathrm{~nm}$ with a relatively narrow distribution in size (Fig. 1d). The islands are distributed on the substrate with a high density of about 150 crystals on an area of $1 \mu \mathrm{m}^{2}$.

XRD analysis (Fig. 2) and HRTEM investigations (Fig. 3) were performed to get structural information on the nanocrystals. An epitaxial relationship between the substrate and top structures was proved. No secondary phase 

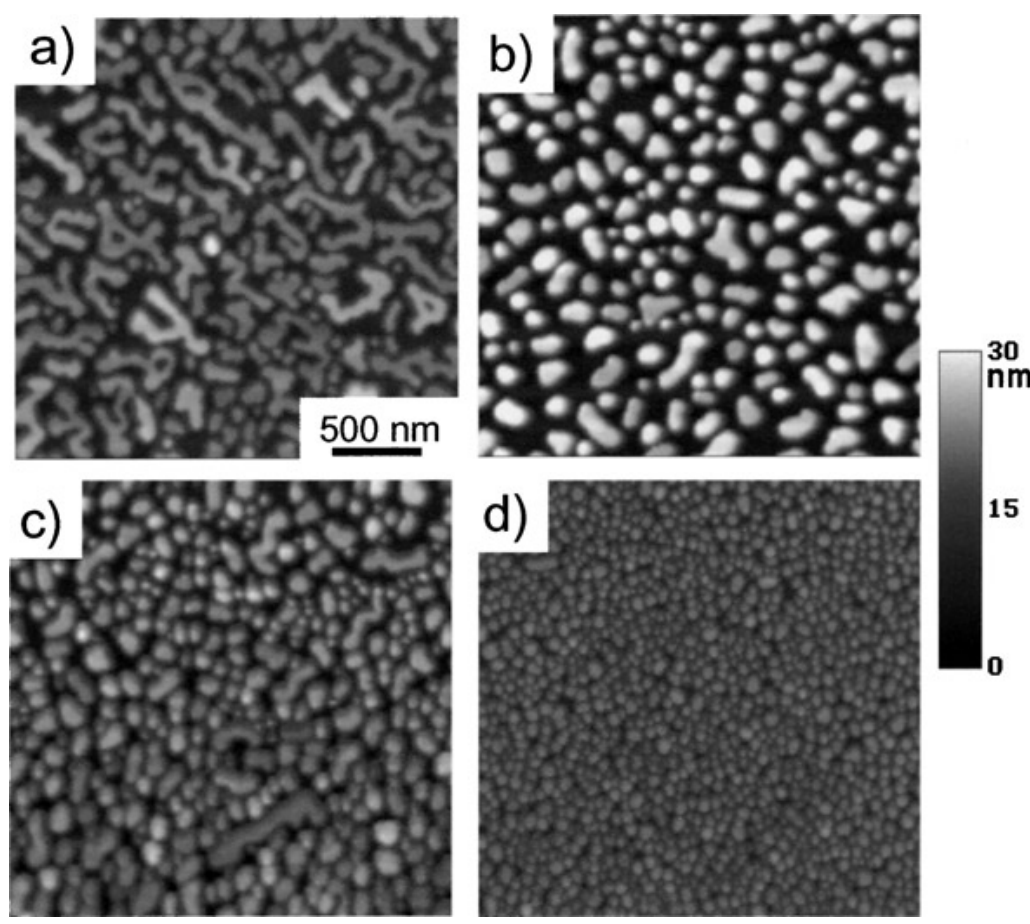

FIGURE 1 AFM images of PZT nanoislands obtained after deposition of (a) 1:20, (b) 1:25, (c) 1:30 and (d) 1:40 diluted PZT precursor.

peak was detected in XRD pattern, confirming the formation of a good quality perovskite phase. Moreover, HRTEM images show that all islands have uniform heights and truncated pyramidal shape with relatively sharp facets that preferably consist of $\{111\}$ and $\{110\}$ planes as lateral surfaces and $\{001\}$ planes as top surfaces [15]. All PZT islands appear as single crystalline with single c-domain orientation. The crystals are free from volume defects, and the only defects are dislocations concentrated along the interface, caused by relaxing the stress induced by lattice mismatch between the substrate and PZT islands.

To investigate the growth mechanism of PZT nanoislands two series of samples were prepared by varying the crystallisation temperature (650$800^{\circ} \mathrm{C}$ for $1 \mathrm{~h}$ ) and time $\left(5 \mathrm{~min}-10 \mathrm{~h}\right.$ at $\left.800^{\circ} \mathrm{C}\right)$. This experiment was done using the initial layers that give $9 \mathrm{~nm}$ high islands after $800^{\circ} \mathrm{C}$ treatment. HRTEM images, shown in Fig. 3, suggest that in the early stage of nucleation 


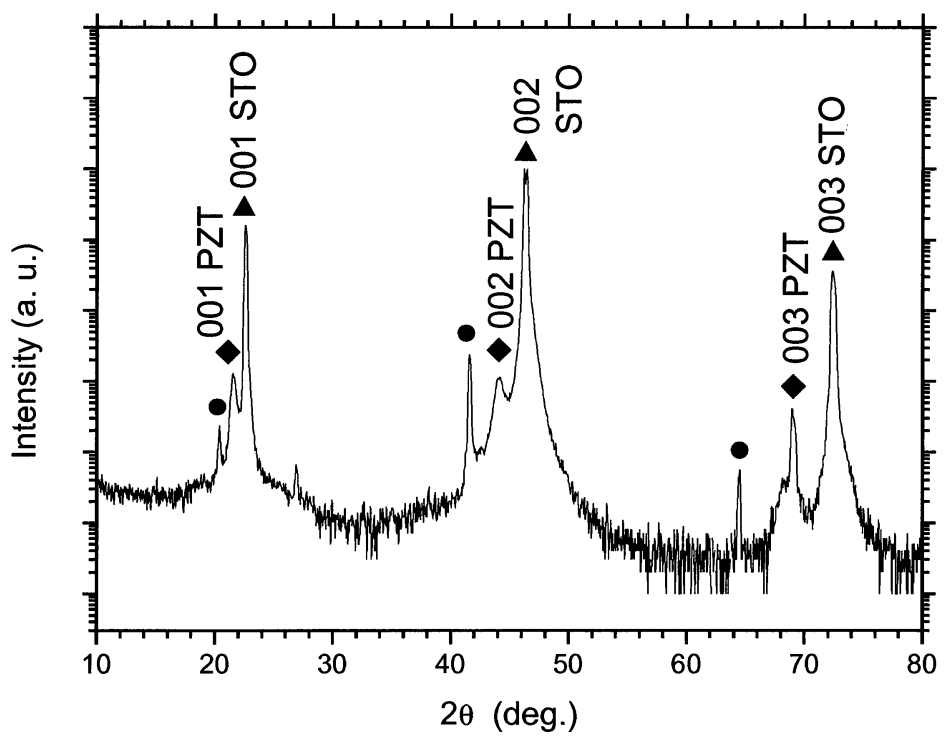

FIGURE 2 X-ray diffraction pattern of PZT nanocrystals. The peaks labelled with circles indicate the substrate peaks originating from the remaining $\mathrm{CuK}_{\beta}$ radiation.

the pre-existing holes penetrate down to the substrate surface and during longer heat treatments $\left(650^{\circ} \mathrm{C}\right.$ for $1 \mathrm{~h}$, see Fig. 3a) the nanoislands migrate on the substrate surface and coalescence into larger irregular structures. Nanostructures crystallised at higher temperatures (e.g., at $800^{\circ} \mathrm{C}$ for $5 \mathrm{~min}$, see Fig. 3b) have better thickness uniformity. However, annealing as long as $1 \mathrm{~h}$ at $800^{\circ} \mathrm{C}$ is needed to form the equilibrium shape and distribution of islands (Fig. 3c). Treatments longer than $1 \mathrm{~h}$ do not lead to significant changes in island morphology, shape and distribution. As it was previously shown, the nanostructure formation is connected with the lowering of the interfacial energy of the film by breaking up the film into islands, which lowers the interface area and consequently the interface energy [11]. However, longer thermal treatments at higher temperature are needed to reach the equilibrium state in which well-defined facets appear.

The ferroelectric properties of individual crystal islands were probed by piezoresponse force microscopy [16]. The islands having a large volume (either with a small lateral size and heights above $25 \mathrm{~nm}$ or thinner with a larger lateral size) show well-developed piezoelectric hysteresis loops [17]. Figure 4 shows the topography and piezoresponse images proving switching 

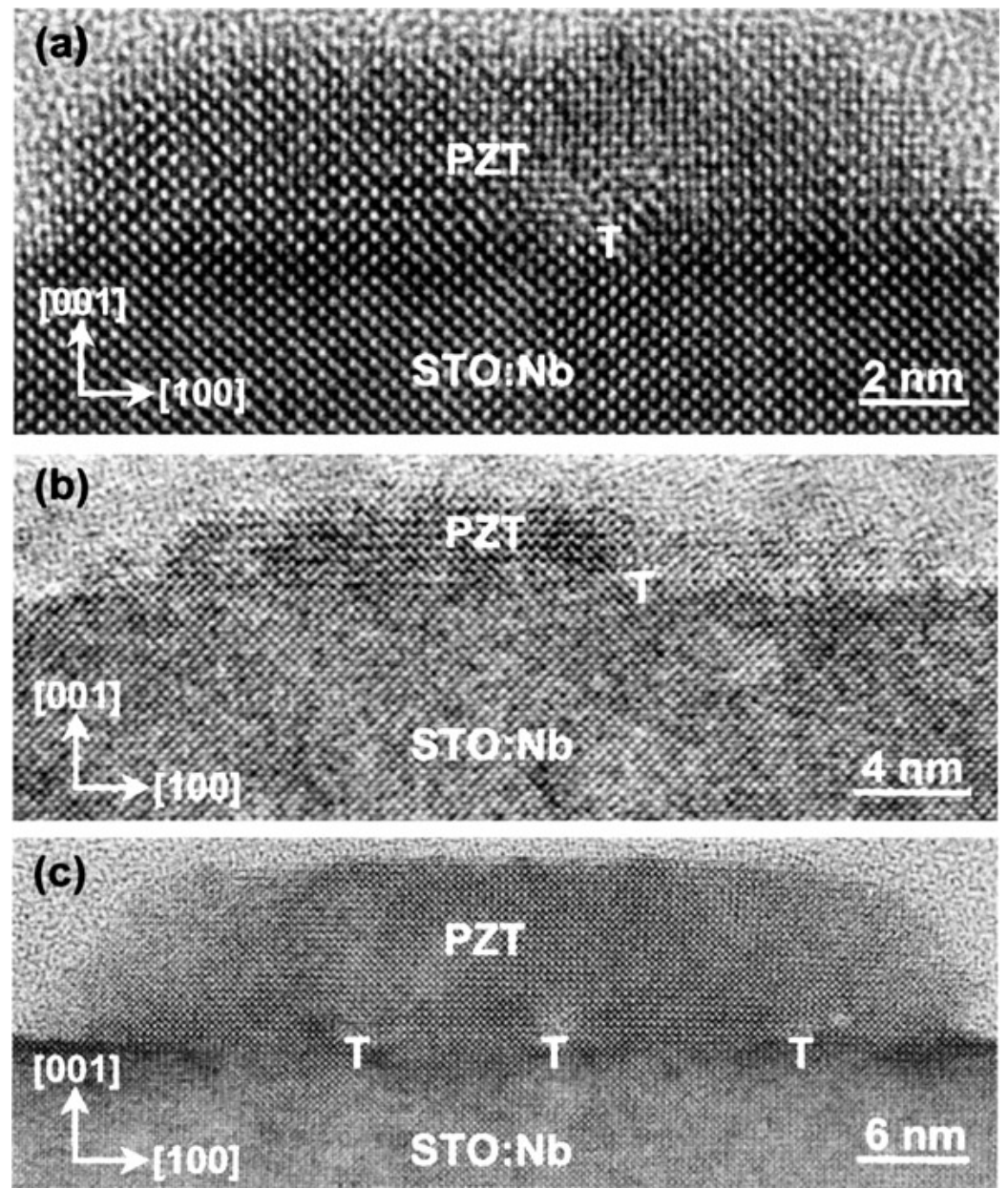

FIGURE 3 Cross section transmission electron microscope image of PZT nanostructures grown on STO:Nb substrate and annealed for (a) $1 \mathrm{~h}$ at $650^{\circ} \mathrm{C}$, (b) $5 \mathrm{~min}$ at $800^{\circ} \mathrm{C}$ and (c) $1 \mathrm{~h}$ at $800^{\circ} \mathrm{C}$.

of polarisation in crystals with a height of $15 \mathrm{~nm}$. The grain marked by the dashed contour was successively polarised negatively (polarisation pointing into the sample, Fig. 4b) then positively (Fig. 4c) by the biased AFM tip. Investigations of the smallest self-assembled structures are under progress in order to establish the minimum size of the epitaxial nano-size crystals that can still be switched. 

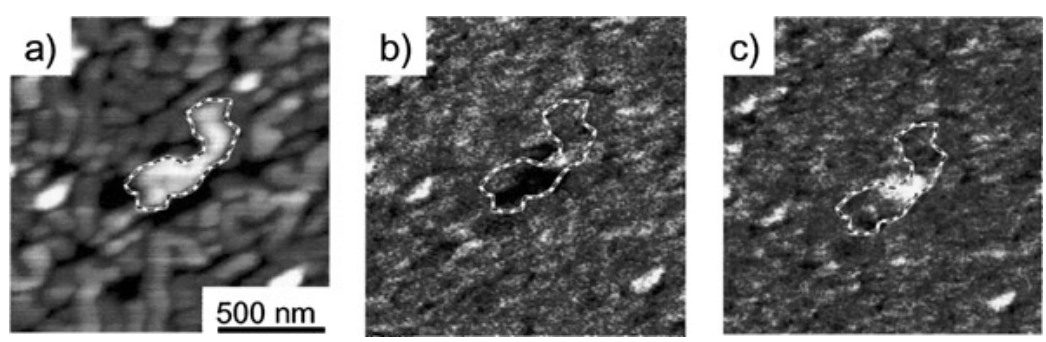

FIGURE 4 Switching of an individual nanocrystal by PFM: (a) topography (z-scale $25 \mathrm{~nm}$ ) and piezoresponse images after applying (b) a negative and (c) a positive dc voltage.

\section{CONCLUSIONS}

Epitaxial nanostructures of ferroelectric PZT have been successfully fabricated onto $\mathrm{Nb}$ doped (001) $\mathrm{SrTiO}_{3}$ single crystals by a self-assembly route that exploits microstructural instability developed in ultrathin films at hightemperatures. The lateral size as well as the height of the nanocrystals can be tuned in a certain range by adjusting the initial thickness of the deposited films and the post-deposition thermal treatment. If the crystallisation process happens at high enough temperatures for sufficiently long times, than all the nanoislands have a well-defined shape with relatively sharp facets that preferably consist of $\{111\}$ and $\{110\}$ planes as lateral surfaces and $\{001\}$ planes as top surfaces. X-ray and TEM confirmed good quality of the epitaxial nanostructures. They are single crystalline, epitaxial, uniform in height and free from any volume defects. Islands of relatively large volume and a height above $15 \mathrm{~nm}$ show ferroelectric properties.

\section{ACKNOWLEDGMENTS}

Part of this work was supported by Volkswagen Stiftung in the Project "Nanosized Ferroelectric Hybrids" under contract No. 5/77737.

\section{REFERENCES}

[1] K. Uchino, Ferroelectric devices (New York, Marcel Dekker, 2000).

[2] R. Waser (ed.), Nanoelectronics and information technology: advanced electronic materials and novel devices (Weinheim, Wiley-VCH, 2003). 
[3] R. Waser, T. Schneller, S. Hoffmann-Eifert, and P. Ehrhart, "Advance chemical deposition techniques-from research to production," Integr. Ferroelectrics 36, 3-20 (2001).

[4] A. Roelofs, T. Schneller, K. Szot, and R. Waser, "Piezoresponse force microscopy of lead titanate nanograins possibly reaching the limit of ferroelectricity," Appl. Phys. Lett. 81, 5231-5233 (2002).

[5] A. Roelofs, T. Schneller, K. Szot, and R. Waser, "Towards the limit of ferroelectric nanosized grains," Nanotechnology 14, 250-253 (2003).

[6] C. Harnagea, A. Pignolet, M. Alexe, and D. Hesse, "Piezoresponse scanning force microscopy: What quantitative information can we really get out of piezoresponse measurements on ferroelectric thin films," Integr. Ferroelectrics 44, 113-124 (2002).

[7] H. Fujisawa, K. Morimoto, M. Shimizu, and H, Niu, "Observations of island structures at the initial growth stage of $\mathrm{PbZr}_{\mathrm{x}} \mathrm{Ti}_{1-\mathrm{x}} \mathrm{O}_{3}$ thin films prepared by metalorganic chemical vapor deposition," Jpn. J. Appl. Phys. 39, 5446-5450 (2000).

[8] W. C. Goh, S. Y. Xu, S. J. Wang, and C. K. Ong, "Microstructure and growth mode at early stage of laser-ablated epitaxial $\mathrm{Pb}\left(\mathrm{Zr}_{0.52} \mathrm{Ti}_{0.48}\right) \mathrm{O}_{3}$ films on a $\mathrm{SrTiO}_{3}$ substrate," J. Appl. Phys. 89, 4497-4502, (2001).

[9] H. Fujisawa, K. Morimoto, M. Shimizu, H. Niu, K. Honda, and S. Ohatani, "Piezopresponse measurements for $\mathrm{Pb}(\mathrm{Zr}, \mathrm{Ti}) \mathrm{O}_{3}$ island structure using scanning probe microscopy," Mat. Res. Soc. Symp. Proc. 655, CC10.4.1-CC10.4.6 (2001).

[10] H. Fujisawa, M. Shimizu, H. Niu, K. Honda, and S. Ohatani, "Observations of domain structure at initial growth stage of $\mathrm{PbTiO}_{3}$ thin films grown by MOCVD," Mat. Res. Soc. Symp. Proc. 596, 321-326 (2000).

[11] A. Seifert, A. Vojta, J. S. Speck, and F. F. Lange, "Microstructural instability in singlecrystla thin films," J. Mater. Res. 11, 1470-1482 (1996).

[12] P. A. Langjahr, T. Wagner, M. Rühle, and F. F. Lange, "Thermally induced structural changes in epitaxial $\mathrm{SrZrO}_{3}$ films on $\mathrm{SrTiO}_{3}$," J. Mater. Res. 14, 2945-2951 (1999).

[13] C. Harnagea, A. Pignolet, M. Alexe, D. Hesse, and U. Gosele, "Quantitative ferroelectric characterization of single submicron grains in Bi-layered perovskite thin films," Appl. Phys. A 70, 261-267 (2000).

[14] C. Harnagea, M. Alexe, D. Hesse, and A. Pignolet, "Contact resonances in voltagemodulated force microscopy," Appl. Phys. Lett. 83, 338-340 (2003).

[15] M. W. Chu, I. Szafraniak, R. Scholz, D. Hesse, and M. Alexe, in preparation.

[16] C. Harnagea, A. Pignolet, M. Alexe, D. Hesse, and U. Gosele, "Quantitative ferroelectric characterization of single submicron grains in Bi-layered perovskite thin films," Appl. Phys. A 70, 261-267 (2000).

[17] I. Szafraniak, C. Harnagea, R. Scholz, S. Bhattacharyya, D. Hesse, and M. Alexe, "Ferroelectric epitaxial nanocrystals obtained by a self-patterning method," Appl. Phys. Lett. (in press). 